\title{
Interactive control of physiological experiments
}

\author{
Jan A. van Alsté, Albert L. Schoute* and Herman B.K. Boom \\ Department of Electrical Engineering, Bio-information Group and * Department of Informatics, Twente University of Technology, \\ P.O. Box 217, 7500 AE Enschede, The Netherlands
}

\begin{abstract}
Physiological and biological experiments often require the imposition of repeated influences on an organ or organic system. Then the experimental protocol requires many repetitive functions with adjustable interval times and magnitudes, which are prepared or dictated by experimental results. To control such an experimental set-up interactively with a small computer we chose a more or less general approach. A special language syntax has been developed for: (i) experiment definition; (ii) experiment composition in advance; (iii) real-time control during the actual experiment; and (iv) registration of the actual protocol parameters. The interactive control was realized using the special language and an interpreter as one of the parallel processes for the experimental control.
\end{abstract}

Experiment control Interactive control Multitasking PASCAL language Physiological experiments Special language

\section{INTRODUCTION}

Many physiological and biological experiments require the imposition of various, repeated influences on some biological organ or organ system. These influences may either be repetitive because of some fundamental periodic property of the experimental subject, or result from the experimenters wish to relate the experimental influences to external repetitive trigger events. In some cases the experimental influences should consist of complexes of chained or synchronous events or processes which have to be repeated many times. Actions could be triggered by some signal derived from the organ or generated by the experimental apparatus.

If, in addition, it is also desired to increase or decrease systematically certain parameters determining or relating the experimental influences from one period to another and during series of periods, then the instrumental hardware required is considerable. The correct manipulation of all controls will be time consuming and considerably error prone.

Clearly computer control greatly facilitates such procedures and can make them interactive for the experimenter. For example, new parameter values may be selected during the experiment after observation of preceding responses.

In our laboratory the pressure generating properties of the left ventricle of the heart are the subject of study. We are particularly interested how in time, relative to the start of systole, the ventricular pressure depends on ventricular volume and flow.

An experimental set-up was built, mainly consisting of a computer-controlled servo piston pump connected to a working isolated left ventricle of a rabbit heart. The experimental results are obtained by measuring the intraventricular pressure during contractions with controlled volume and volume changes, providing the desired flow patterns. This occurs under a great variety of conditions governed by the occurrence and magnitude of stimulation 
time, opening and closing time of valves, time and speed of pump piston movement, automatic calibration procedures, and sampling time of several signals [1]. The experiments are therefore complex, and more than 60 control parameters must be selected for every ventricular beat. Since the primary task of the experimenter is the design of the experimental protocol followed by the interpretation of the experimental results - and not the translation of the protocol into dial settings we felt that interactive computer control could be an important tool in this type of experiment. This control should be such that:

(i) The experimenter can unambiguously specify any experimental action to be performed; and

(ii) The program can be interrupted at any time if the situation requires changes or special attention;

(iii) It is possible to specify any new experimental program while the experiment is actually running.

This can be done by either naming previously defined and prepared sequences of control actions or - prompted by the actual results - by composing new series. Finally in such a flexible environment for experimentation, protection against unnecessary mistakes and proper recording of the actual course and results of the experiment, is vital.

We needed a (quasi) parallel processing system for the interactive computer control, which, on the one hand supports the control processes for the specific experimental actions and on the other hand an 'interpreter' process for the user interaction. This relieves us of the disadvantages of previous software control systems [2]. The interpreter was fitted to a special experiment language that was developed for:

(i) experiment definition;

(ii) experiment composition in advance;

(iii) real-time control during the actual experiment; and

(iv) logging of the actual experimental protocol and its parameters used.
Although the interactive experiment-control software was specially designed for the abovementioned experiments, its design is more general and may be used in other experiments of a similar nature. In this paper we describe the realization of interactive control emphasizing the general design considerations. The system performance and limitations are considered and conclusions will be stated.

\section{INTERACTIVE CONTROL}

The basic philosophy in the design of the interaction between computer and the experimenter was to relieve the latter of low-level control and instrument-dependent timing aspects.

An experiment, consisting of sequences of actions, needs to be specified only at such a level, that the experimenter can think in terms of fundamental actions within the experiment. These sequences should be carried out on demand. Response to expected, but unplanned problems, should be automatic and in a preprogrammed way.

The actual control parameters used should be specified and changed interactively. The conversion of the parameters into the appropriate computer actions is carried out by the computer program. Of course automatic precautions against undesired control actions should be taken to prevent damage to the biological preparation or the apparatus, whether they are due to wrong commands or to parameters that stray from their normal range.

Choosing for interactive control of experiments may mean that the logging of the actions taken is essential, since changes in the protocol may take place at a substantially higher rate and experiments will follow a more complicated course.

In order to be able to meet all the requirements for this type of experiment it was necessary to develop a special language for experiment description and another for experiment control.

\subsection{The experiment description language}

The experiment description language is designed for the unique description of the actions to be 
performed during the experiment and the logging of the experimental actions realized. This language specifies an experiment as a sequence of basic experimental cycle conditions (BECC), (in our case consisting of one heart beat), with their corresponding parameter settings. The syntax of such a specification language for our experiments is shown in Fig. 1, and conforms with appendix D of Jensen and Wirth [4].

We can name a specified sequence of BECC's for our experiments by means of an identifier in the following way:

The defined sequence VOLRAMP consists of

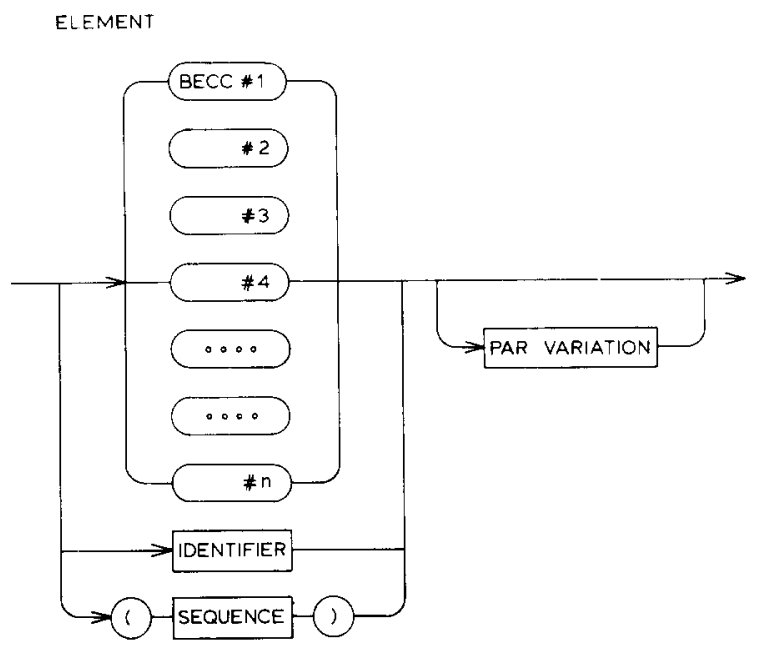

SEQUENCE
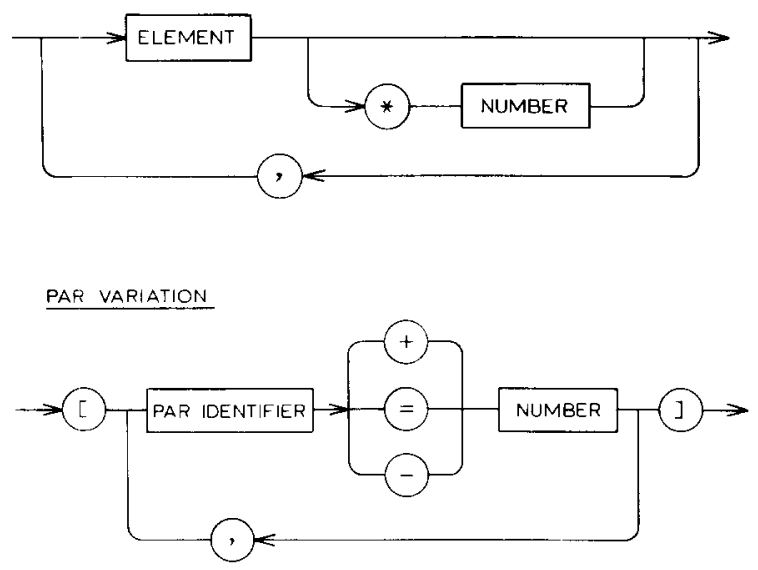

Fig. 1. Syntax diagram of the experiment description language.
VOLRAMP $=$ EDPRES[PRES $=2.0] \star 1 \emptyset$, RAMP

Fig. 2. An example of a simple experiment definition.

10 basic heart cycles of type EDPRES with the parameter setting PRES $=2.0$, followed by one basic heart cycle of the type RAMP.

This example is presented in Fig. 2.

As shown, the sequences consist of elements with possible attributed parameter settings that describe one BECC, separated by commas and possibly followed by a repetition factor. In this way runs of a specified (arbitrary) number of identical or different cycles can be assembled.

The elements of a sequence definition itself may consist of basic (reserved) identifiers describing one specific BECC, here a heart cycle, (implying specific control conditions). They may consist of already defined sequence identifiers or alternatively of an expression between brackets that conforms to the SEQUENCE syntax. In this way earlier defined sequences can be used to define more complex sequence structures. Therefore in our specific application the syntactic elements EDPRES and RAMP, as shown in Fig. 2, are reserved identifiers for two different heart cycle conditions.

The experimental parameters, like PRES in our example, can receive their value by default but may be changed. The parameter variation block PAR VARIATION of the syntax diagram of Fig. 1 shows the possibilities of specifying a new parameter value $(=)$, extended with an optional automatic increment $(+)$ or decrement $(-)$ every time the element concerned is executed.

A more elaborate example of an experiment definition is given in Fig. 3. Here STEADY represents a sequence of 20 identical heart beats. FLOWARRAY specifies an experimental sequence in which after. 20 identical heart beats (STEADY), 5 times a combination of one heart beat, with increasing parameter FLOW and ten equal beats with PRES $=1.0$ are specified.

I SOVOL = EDPRES[PRES $=2.0]$

STEADY $=$ I SOVOL $* 26$

FLOWARRAY $=5$ TEADY, $(\operatorname{RAMP}[$ FLOW $+1,0], \operatorname{EDPRES}[$ PRES $=1,0] * 16) \star 5$

Fig. 3. An example of an experiment definition. 


\subsection{The experiment control language}

The experimental description language is integrated in a command language for the interactive control of the experimental set-up. The commands are able to define experiments, to actually start and stop them, to set the time, to manage an experiment definition library, etc.

Some of the commands we use are shown in the common syntax diagram of Fig. 4. Here the command SETTIME sets the internal computer realtime clock. The command GO initiates the start of the basic cycle control. This cycle consists of the minimum experimental actions that are necessary to keep the biological preparation in good condition and must therefore go on permanently. Executed experimental sequences will invoke control actions on top of this minimum. The system will return to the basic control after an experimental sequence has been stopped or completed, in the case of another sequence not being started. New experiment sequences are defined using the MAKE command and experiments are started or stopped using $\mathrm{DO}$ and $\mathrm{HO}$.

Experiment sequences defined with MAKE are stored in the experiment library. Library commands exist which show experiment definitions on a terminal, give the actual parameters that are asked for, or delete specified experiments as well as any compound experiment containing the specified one. The delete command is for making the involved memory space available again.

\section{COMMANDS}

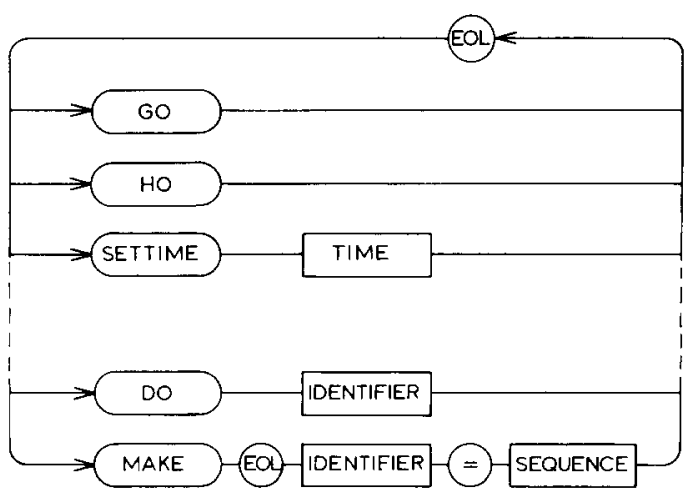

Fig. 4. Syntax diagram of the experiment control language.

\section{THE CONTROL SOFTWARE}

\subsection{Multitasking}

During experiments the tasks of the control computer consist of real-time process control, communication with the experimenter, and management of the library of experiment definitions. To realize these various tasks simultaneously we started from a package of basic routines for parallel processing on a single processor system already available to us [3]. This package enables multitasking (multiprogramming) in a PASCAL environment. A number of extensions were made to make real-time process control possible. It is essential for multiprogramming that the state of execution of a number of tasks is simultaneously preserved in memory so that the execution of these tasks when can be restarted after they have been suspended.

All the necessary routines (called processes) in our application are brought into action in this way when required. The different actives processes are of course served in turn by the single central processing unit (CPU) of the control computer. Queues are used for chained processes that are 'passivated' and for some reason have to wait their turn. These processes are inactive and not served by the CPU. They are released from the queue in a first-in first-out manner and again reactivated by other processes which remove the reason for waiting.

Queues are in particular connected to so-called semaphores. Processes can also deactivate themselves from real-time control, until a specific point of time using a procedure called WAIT. These deactivated processes are placed in a queue called WAITERSLIST (sequenced according to the 'wake-up' time) and will be activated in due course by means of a clock interrupt-driven process. Activation of a process means placing of the process in the CPU-ready queue. The actual time is indicated by a system variable in which the clock interrupts are counted.

For the real-time control two run-priorities are introduced with corresponding ready-queues for the CPU. All processes activated by the clock process after a WAIT are assumed to perform 


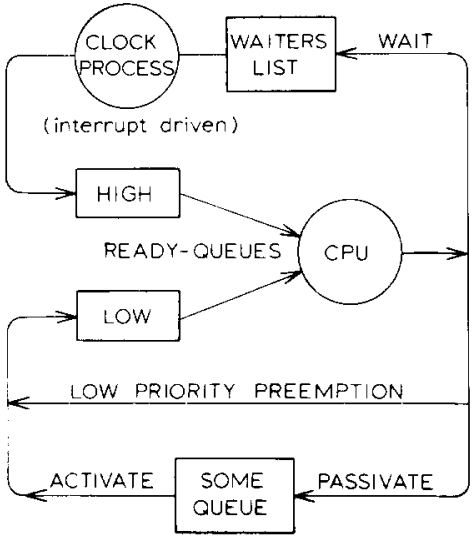

Fig. 5. Process flow.

time-critical actions and are treated on a high-priority basis. Processes with high run-priority will preempt low priority processes, but a high-priority process will remain on the CPU as long as it is required. It is in this respect that conflicts between processes, competing for the CPU in the same (real) time, may occur. The process-flow is summarized in Fig. 5.

\subsection{Command interpreter}

An important aim of the control software is interactive experimenting. Therefore a command interpreter process has been' introduced, so that experiments can be conducted with the use of a terminal. After entering a command in the terminal, the command interpreter analyses the character string and checks if the syntax is correct and then starts the required actions. The use of the command interpreter has two aspects:

(i) It presents the possibility of direct interaction with the experiment by means of start or stop commands;

(ii) It provides the possibility of preparing 'programs' in advance to control the experiments. With respect to aspect (ii) the command interpreter serves as a compilers, which translates the controlling programs into an appropriate lower level code.

The experiment language as described earlier may be considered as an expression language in which, according to the syntax rules, elements of the type SEQUENCE can be nested in any arbitrary way. Multiple occurrences of the same sequences of basic experimental cycles need to be defined only once. This makes the 'code' which is generated by 'compilation' of the sequence definitions compact. The resulting code has a tree structure in which common subtrees are stored. The data used during execution such as repetition counters and the positions in the tree structure must be allocated stackwise.

The tree structure is extended for the automatic parameter incrementing and decrementing with pointers to parameters that have to be changed during execution. Every field of the tree is extended with two pointers. One points to an array with new parameter values and the other to an array with increment or decrement values.

\subsection{Experimental library management}

The number of experiment definitions used in one day's experiments exceeds the capacity of the work memory. Therefore a permanent library was created on a mass storage device, where 'standard' experiment definitions could be retained. Newly entered experiment sequences are expanded forming their structure to test their syntax correctness and the uniqueness of their identifier before installing them in the library. For execution of the experiment the definitions concerned are taken from the library and expanded into their tree structure.

The efficiency of the library use during experimentation is improved by copying its directory and also the necessary experiment definitions into the work memory.

\subsection{The control processes}

The control tasks are split up into separate processes so that each process corresponds to a sequence of conceptually related experimental actions.

In this way programming of the different control actions can be done independently of each other. The different control processes, however, need to be synchronized. 

tion:

We can distinguish two types of synchroniza-

(1) Semaphore activation: an inactive process (waiting in a semaphore queue because of a passivate-operation) is made active by another process (due to an activate-operation on the semaphore queue). The activation need not be immediate as it is only guaranteed within ' finite time'. In practice, however, the processing capacity may be sufficient to assure quick responses.

(2) Real-time activation: a process can delay itself until a specific instant in real time by means of the WAIT-procedure. Processes can synchronize their actions relative to a common time base by passing information on timing, so that 'time shifts' between processes are avoided.

A master-slave relationship between processes is desirable for synchronization. The master can activate his slaves as desired and can pass timing information, relative to which the slave processes synchronize their actions. In our application one of the control processes (controlling the time of stimuli of the heart cycle) provides the central control mechanism and functions as a master process. It can handle all the essential control to be done continuously, and performs the organizational task of activating the other (slave) processes according to specified conditions. The slaveprocesses in our applications handle optional cases of experiment control (they are active only within one cycle) or treat special cases.

\section{CONCLUSIONS}

The software control system has now been in use for more than a year and we found it very suitable to our needs.

The advantages of this system for the programmer are:

- Separate description of processes, making it easy to add new processes or change existing ones.
- The use of the computer language PASCAL for all programs.

Important features for the experimenter are:

- A formal, quantitative description of individual experiments in terms of the structure of the experiment language.

- The standardization of experiments.

- Independent specification of real-time events for different processes (this avoids specification of intervals between uncorrelated events).

- The interactive control due to the command interpreter process.

- The ease of preparing experiments in advance and during operation due to the experimental language.

- The try-out possibility of new experiment definitions using dummy hearts, so the actual experiments can be more successful.

- The high reproducibility of control actions due to digital management.

The versatility of the computer control gives the experimenter the opportunity to pay attention to the execution of the experiments.

The control actions normally take only several milliseconds. Due to the light load on the CPU, the interference of control actions is limited and the accuracy obtained is within 1 millisecond. Problems will arise when the load on the system grows, because of control-action contention and CPU-overhead. Measures taken to solve problems as, for instance, special scheduling and low level programming, tend to upset, in one way or another, the flexibility with which the interactive control can be realized.

The general software approach presented in this paper, is a very attractive way of real-time process control in the case of processing requirements which are not too heavy. 


\section{REFERENCES}

[1] S.R. Vaartjes, J.A. van Alsté and H.B.K. Boom, Active resistance during left ventricular contraction, in: Proc. 35th Ann. Conf. Engineering in Medicine and Biology, p. 142 (Philadelphia, PA, 1982).

[2] J.A. van Alsté, E. Baarsma and J.P. van Dijk, A small software control system for (physiological) experiments, in: Proc. DECUS pp. 239-240 (Monte Carlo, 1979).

[3] C. Bron, PASCAL used for operating system implementation, in: Proc. PASCAL conference (Gothenburg, 1980).

[4] K. Jensen and N. Wirth, PASCAL user manual and report, Appendix D (Springer Verlag, Berlin, New York, NY, 1978). 